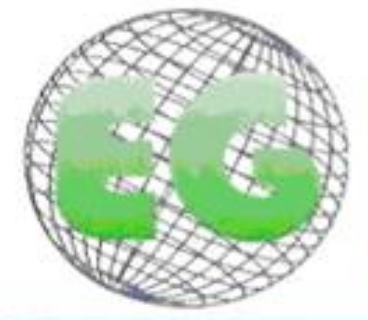

ISSN 1695-6141

www.um.es/egloball

\title{
CLÍNICA
}

\section{Complicaciones presentadas en pacientes mayores de 65 años ingresados por fractura de cadera en un hospital andaluz de tercer nivel}

Complications presented in patients over 65 years admitted for hip fracture in an Andalusian tertiary hospital

\section{*López-Hurtado, Felipe ${ }^{* *}$ Miñarro del Moral, Rosa María ${ }^{* * * A}$ Arroyo Ruiz,Verónica ${ }^{* * * *}$ Rodríguez-Borrego, María Aurora}

*Departamento de Enfermería. Universidad de Córdoba. E-mail: felohur@yahoo.es **|MIBIC/Hospital Universitario Reina Sofía/Universidad de Córdoba ***Hospital Universitario Reina Sofía ****Departamento de Enfermería. Universidad de Córdoba. IMIBIC/Hospital Universitario Reina Sofía/Universidad de Córdoba. España.

Palabras Clave: Fractura de cadera; complicaciones; ancianos; 65 años; CMBD (Conjunto Mínimo Básico de Datos de Andalucía); alta.

Keywords: Hip fracture; complications; elderly; 65 years old; Andalusian Minimum Data Set Basic Minimum Data; discharge

\section{RESUMEN}

Objetivo: Conocer las complicaciones que se han producido en pacientes mayores de 65 años, ingresados en un hospital andaluz de tercer nivel durante el año 2012, con diagnóstico principal de fractura de cadera.

\section{Metodología:}

Diseño: Se realizó un estudio descriptivo transversal.

Ámbito, periodo y sujetos de estudio: Pacientes ingresados en el año 2012 mayores de 65 años con diagnóstico principal de fractura de cadera.

Variables principales: Morbimortalidad recogida en el CMBD, analizada en función de otras variables que influyen en su aparición según la bibliografía (edad, sexo, comorbilidad, etc). Análisis estadístico de las variables con comparación de medias y proporciones con los programas estadísticos Epidat y GStat de uso libre.

Resultados: La media de edad fue de 83,16 $(6,68)$ años, y de estancia 10,2 días. La mortalidad fue del $4,99 \%$. Las fracturas extracapsulares fueron más frecuentes que las intracapsulares, $64,24 \%$ y $35,76 \%$ 
respectivamente. La estancia media preoperatoria, 3,5 días. El tratamiento más frecuente la osteosíntesis, con un 62,6\%. La complicación más frecuente, excluyendo el éxitus, "complicaciones quirúrgicas- vías urinarias". El total de pacientes complicados fue del 8,7\%, incluyendo los fallecidos. El sexo masculino, la edad avanzada y la demora quirúrgica se asocian con aparición de complicaciones. Los porcentajes más altos de complicación se dieron en pacientes con tres comorbilidades asociadas.

Conclusión: La presencia de complicaciones en el período de hospitalización de pacientes ancianos ingresados por fractura de cadera se relaciona con sexo masculino, edad avanzada, demora quirúrgica y mayor número de comorbilidades.

\section{ABSTRACT}

Purpose: To know the complications that occurred in patients over 65 years admitted to an Andalusian tertiary hospital during 2012 with a primary diagnosis of hip fracture.

\section{Methodology:}

Design: Cross-sectional study.

Scope, period and cohort: Patients admitted in 2012 over 65 years with a primary diagnosis of hip fracture.

Main outcomes: Morbidity and mortality collected in the Andalusian Minimum Data Set , analyzed in terms of other variables that influence their occurrence according to the literature (age, sex, comorbidity, etc).

Instruments: Statistical analysis has been done with Epidat and G-Stat, statistical free software, to get and compare means and proportions of the variables.

Results: The mean age was $83.16(6.68)$ years old. The mortality rate was $4.99 \%$. The average length of stay was 10.2 days. Extracapsular fractures were more frequent than intracapsular ones, $64.24 \%$ and $35.76 \%$ respectively. The average preoperative stay was 3.5 days. The most frequent treatment was osteosynthesis, with $62.6 \%$. The most common complication, excluding exitus, was "surgical urinary tract complications". Total complicated patients was $8.7 \%$, including the deceased. Male gender, older age and surgical delay was associated with development of complications. The highest percentages of complications occurred in patients with three comorbidities.

Conclusion: The presence of complications during the period of hospitalization of elderly patients admitted for hip fracture is associated with male gender, older age, surgical delay and more comorbidities.

\section{INTRODUCCIÓN}

La fractura de cadera, en los pacientes ancianos, es la causa más frecuente de ingreso en el hospital en los servicios de traumatología y ortopedia, y son los pacientes que mayor morbilidad y mortalidad presentan en estos servicios $^{(1,2)}$.

La incidencia de las fracturas de cadera aumenta con la edad, llegando a doblarse para cada década a partir de los 50 años ${ }^{(3)}$, si bien otros autores consideran que su incidencia sigue un patrón exponencial de los 60 años hasta la edad de $84{ }^{(4)}$.

Los últimos datos del censo, publicados por el Instituto Nacional de Estadística, muestran que en 2011 la esperanza de vida, al nacimiento en España, superaba los 82 años, y alcanzaba los 85 para las mujeres ${ }^{(5)}$. En Andalucía ya hay un $15 \%$ de ciudadanos con más de 65 años, y un $17 \%$ en España ${ }^{(6)}$, lo que muestra el envejecimiento de la población, con el consiguiente aumento del riesgo de sufrir fracturas de cadera. Esto acentúa la importancia en términos de salud y gasto sanitario de esta lesión para el conjunto de la sociedad. Tanto es así que la 
Consejería de Salud de la Junta de Andalucía, dentro del Mapa de Procesos Asistenciales Integrados, para ordenar y coordinar la asistencia sanitaria mediante el análisis secuencial de los distintos procesos por los cuales el ciudadano acude a los centros sanitarios, seleccionó la "Fractura de cadera en el anciano" como uno de los procesos que representa una proporción importante de actividad dentro del Sistema Sanitario Público de Andalucía, dedicándole un Proceso Asistencial Integrado específico ${ }^{(7)}$.

La fractura de cadera en el anciano supone un problema importante para el sistema sanitario por su alta prevalencia y morbimortalidad ${ }^{(7)}$. Entre el 2 y el $7 \%$ de los pacientes fallecieron durante la fase hospitalaria aguda, entre el 6 y el $12 \%$ durante el mes posterior y entre el 17 y el $33 \%$ al cabo del primer año tras la fractura. Puede estimarse que, en España, de los casi 40.000 pacientes que sufren anualmente una fractura de cadera, fallecerán unos 10.000 durante los doce meses posteriores a la misma. Respecto a la morbilidad, indican que entre el 15 y el $30 \%$ de los pacientes presentan complicaciones graves durante la fase aguda, aunque la frecuencia y tipo de las mismas presentan una amplia variabilidad entre diferentes hospitales. Las complicaciones menores como la anemia leve, el estreñimiento o las formas leves de delirium pueden alcanzar cifras mucho más elevadas, pues se ha cuantificado una media de 2 complicaciones por paciente ${ }^{(8)}$.

Las complicaciones son más frecuentes en personas de ciertas características como: edad más avanzada, sexo masculino, mala función previa, sometidos a anestesia general, necesidad de transfusión, alto grado en la escala de la American Association of Anaesthesiologists (ASA), y en los que padecen ciertas enfermedades (insuficiencia cardíaca, diabetes, EPOC, insuficiencia renal, neoplasias, malnutrición, deshidratación, hepatopatías y secuelas de ictus, entre otras) así como en aquellos en los que se retrasa la cirugía. Parece existir una frecuencia mayor, en varones, de algunas de las complicaciones como neumonía, tromboembolismo pulmonar o úlcera de estrés ${ }^{(8)}$.

Un estudio reciente ha demostrado que el manejo de los pacientes con fractura de cadera durante su fase aguda, siguiendo las vías clínicas estandarizadas, reduce notablemente la frecuencia de algunas complicaciones, concretamente trombosis venosa profunda, úlceras por presión, infección urinaria e infección de la herida quirúrgica. Las complicaciones mayores no quirúrgicas pueden ser la causa de la muerte en más de la mitad de los fallecimientos que ocurren en el primer mes del posoperatorio ${ }^{(8)}$.

Según la Clasificación Internacional para la Seguridad del Paciente, se entiende como "Complicación" al trastorno del paciente que surge durante el proceso de dispensación de atención sanitaria, sea cual sea el entorno en el que se dispensa, o la enfermedad o lesión que surge a raíz de otra enfermedad y/o intervención asistencial, y como "Incidente relacionado con la seguridad del paciente" al evento o circunstancia que ha ocasionado un daño innecesario a un paciente o podría haberlo hecho ${ }^{(9)}$.

Por su parte, la "Seguridad del Paciente" se define como la ausencia de daño evitable a un paciente durante el proceso de la atención sanitaria, o la reducción a un nivel mínimo aceptable de riesgo de sufrir daño innecesario en el curso de la atención sanitaria. El "nivel mínimo aceptable" hace referencia al nivel de conocimiento actual, los recursos disponibles y el contexto en que se produce la atención frente al riesgo 
de no tratamiento u otro tratamiento. "Evento adverso" (o Efecto Adverso) (EA) es un incidente que causa daño al paciente $(9,10,11,12,13)$.

En base a lo anterior, las complicaciones de la fractura de cadera parecen efectos adversos (EAs) que se podrían evitar.

Los EAs ligados a la asistencia son un problema frecuente en la práctica clínica en cualquier nivel de cuidados. Los pacientes que ingresan para una intervención quirúrgica tienen en torno al $30 \%$ de riesgo de sufrir un EA ${ }^{(14)}$, porcentaje que puede reducirse de forma significativa con medidas preventivas que tienen ya evidencia científica ${ }^{(15)}$. Según el Estudio Nacional sobre los Efectos Adversos ligados a la Hospitalización en España (ENEAS 2005), los pacientes mayores de 65 años tienen 2,5 veces más riesgo de sufrir un EA que otro paciente de menor edad, y se ha constatado la asociación entre la alteración de las barreras naturales de entrada (sondas y cateterismos, de las que serán portadores la mayoría de los pacientes que nos ocupan) con la incidencia de la infección nosocomial ${ }^{(14,16)}$.

Las bases de datos administrativas se encuentran entre los métodos propuestos por la Organización Mundial de la Salud para conocer la magnitud de los EAs ${ }^{(17)}$. El Conjunto Mínimo Básico de Datos de Andalucía (CMBD Andalucía) es un registro administrativo que contiene un conjunto de variables clínicas, demográficas y administrativas que resumen lo acontecido a un usuario en un episodio de asistencia hospitalaria. Proporciona información básica sobre el usuario, sobre el centro y unidad que lo atiende y sobre su proceso asistencial ${ }^{(18)}$.

En Andalucía, desde el 1 de enero de 2010, es obligatoria la cumplimentación de la "condición de presente al ingreso o al inicio del contacto" (POA - present on admission) tras cada uno de los códigos diagnósticos del CMBD, lo que permite, entre otras cosas, mejoras en la detección de problemas de seguridad de los pacientes ${ }^{(18)}$.

En el contexto descrito surge la pregunta de cuál es el mapa/evolución/situación de la frecuencia y/ o características de complicaciones en pacientes mayores de 65 años ingresados por fractura de cadera en un hospital de tercer nivel en el occidente andaluz. La información contenida en las bases de datos puede ofrecer una fotografía de la situación que permita plantear las bases para establecer protocolos de seguridad del paciente ante complicaciones, efectos adversos evitables, intervención que justifica por sí misma el presente estudio.

Se diseñó un estudio con los siguientes objetivos:

\section{General:}

Conocer las complicaciones que se han producido en pacientes mayores de 65 años ingresados en un Hospital de tercer nivel del occidente andaluz, durante el año 2012, con diagnóstico principal de fractura de cadera.

\section{Específicos:}

1. Identificar las presencia de complicaciones por episodio* de hospitalización. 
2. Enunciar factores relacionados con la presencia de complicaciones y EAs.

*Se entiende por episodio aquellos ingresos con fecha de alta en el CMBD. (Conjunto Mínimo Básico de Datos de Andalucía)

\section{METODOLOGIA}

Se realizó un estudio descriptivo transversal, cuyo ámbito de estudio fue la base de datos del Conjunto Mínimo Básico de Datos de Andalucía (CMBD) de un hospital de tercer nivel en el occidente de la Comunidad Autónoma Andaluza, durante el año 2012.

El objeto de estudio fueron los episodios registrados en el CMBD en pacientes mayores de 65 años, con diagnóstico principal de fractura de cadera y alta en el 2012. Las variables dependientes son las complicaciones (CCS y MSC) y los exitus.

Las independientes son la edad, sexo, tipo de fractura (extracapsular o intracapsular), tipo de intervención (osteosíntesis, prótesis parcial, prótesis total o conservadora), estancia (no de días), tiempo de demora quirúrgica y comorbilidad asociada (según índice de Elixhauser).

Se utilizaron como fuente los programas Access (de Office) y Base (Openoffice) para el procedimiento de recogida de datos.

Las complicaciones registradas en el CMBD se recogieron cruzando estos datos con el Software de Clasificaciones Clínicas (CCS), herramienta para la agrupación de diagnósticos y procedimientos a pacientes en un número manejable de categorías clínicamente significativas. CCS ofrece a los investigadores la capacidad de agrupar procedimientos y condiciones sin tener que clasificar a través de miles de códigos CIE (Clasificación Internacional de Enfermedades). De todos los diagnósticos que recoge la CIE el estudio utilizó los incluidos en las categorías CCS 237 Complicación de dispositivo, implante o injerto y CCS 238 Complicaciones de procedimientos quirúrgicos o atención médica.

Otra manera de obtener complicaciones fue a través de los indicadores iCMBD que ofrece el Ministerio de Sanidad y Consumo. La información incluye varias familias de indicadores: frecuentación, tasas de realización, mortalidad, complicaciones, entre otros, con diferentes ejes de análisis (descriptivo, serie temporal, mejores resultados, etc.), para las distintas variables de clasificación y/o filtro del CMBD (geográficas, demográficas, clínicas, de episodio).

Las variables edad, tipo de fractura, tipo de intervención, estancia y tiempo de demora quirúrgica fueron calculadas y/o extraídas de los datos que ofrece el CMBD, así como la variables sexo, comorbilidad y exitus.

Para la determinación de comorbilidades se han utilizado las incluidas en el índice de Elixhauser siguiendo los criterios del Ministerio, que ofrecen una aproximación a la severidad del caso atendiendo a la presencia de comorbilidades no agudas no relacionadas con el diagnóstico principal y que en estudios previos se han mostrado como influyentes en la duración de la estancia, la mortalidad, y el gasto (entre otros). 
Se realizó un análisis descriptivo de todas las variables estudiadas, con frecuencias absolutas y relativas para las cualitativas, y media y desviación típica para las cuantitativas. Se aplicó la Prueba de Ji-cuadrado para tablas $2 \times 2$ o la Prueba exacta de Fisher, y la Prueba de Ji-cuadrado para tablas hxk, para comparar proporciones, y la Prueba $t$ de Student o ANOVA de un factor para comparación de medias. La estimación de los valores se hizo con un intervalo de confianza del 95\%.

En cuanto a las limitaciones del estudio, el CMBD puede presentar errores porque el informe clínico en el que se recogen los datos puede estar incompleto o por error en la codificación; no obstante, el control de calidad aplicado al CMBD en Andalucía muestra resultados satisfactorios. Además, habría que contar con la limitada capacidad del CMBD para diferenciar los problemas de seguridad de aquellas complicaciones derivadas de la naturaleza de la enfermedad del paciente.

El proyecto fue presentado al Comité de Ética de la Investigación de la Provincia de referencia. La persona responsable del manejo de la base de datos administrativa entregó al Investigador Principal los datos anonimizados, y en todo caso se respetó la Ley Orgánica 15/1999, de 13 de diciembre, de Protección de Datos de Carácter Personal.

\section{RESULTADOS}

El número total de pacientes ingresados con fractura de cadera e identificados a partir del CMBD en 2012 fue de 481; 107 hombres (22,25\%); 374 mujeres (77,75\%).

La media de edad de los pacientes estudiados fue de $83,16(6,68)$ años, en un rango de 65-106. Por sexos, la edad de media en mujeres fue de $83,52(6,54)$ y en hombres $81,88(7,03)$. El $31,6 \%$ de las fracturas tiene lugar en el grupo de edad de 80 a 84 años, seguido del grupo de 85 a 89 , con un $26 \%$.

El ingreso hospitalario fue urgente en el $89,81 \%$ de los casos. Del total, el $94,18 \%$ regreso a su domicilio y un $4,99 \%$ fue exitus. La estancia media fue de 10,2 días.

El diagnóstico más frecuente en un 45,32\% de los casos fue Fractura de sección trocanterea neom de cuello femur cerrada; seguido de otras fracturas intracapsulares del cuello de femur-cerrada con un 34,93\%; con una diferencia considerable con el tercero en el ranking, 9,15\% la fractura de sección subtrocanterea del cuello femurcerrada. Del total de fracturas, el $64,24 \%$ fueron extracapsulares, y el $35,76 \%$ intracapsulares. La estancia media preoperatoria fue de 3,5 días.

El tratamiento más frecuente fue la reducción cerrada de fractura con fijación interna en fémur $56,96 \%$ de los casos, seguida de sustitución parcial de cadera con un $28,69 \%$. Se realizó osteosíntesis en el $62,6 \%$ de los casos intervenidos quirúrgicamente.

Del total de pacientes estudiados, sólo un 3,74\% presentó complicaciones CCS. De entre ellas se enumeran algunas: Complicaciones quirúrgicas-vías urinarias (que es la más frecuente); otra hipotensión iatrogénica; sobrecarga circulatoria asociada a transfusión; complicaciones quirúrgicas-cardiacas; o seroma que complica un procedimiento. 
Según los datos recogidos a través del Ministerio de Sanidad y Consumo, se produjeron cuatro complicaciones más que no eran recogidas por el CCS.

El número máximo de complicaciones por episodio fue de dos.

En el análisis bivariante se observa mayor proporción de hombres entre los pacientes que presentan complicaciones, con una significación de 0.011. Del total de hombres, fallecieron un $7,48 \%$, frente a un $4,28 \%$ de mujeres. La media de edad entre los complicados fue de 86,07 $(7,31)$ mostrando diferencia por edad, con una significación de 0,003 . No se encontraron diferencias significativas por grupos de diagnóstico. Por su parte hay presencia de diferentes porcentajes de complicados en función de los procedimientos, con un $8,7 \%$ de complicados independientemente del procedimiento. La media de estancia entre pacientes con complicaciones $(15,93)$ y la demora quirúrgica $(4,03)$ presentan una relación significativa entre los complicados de 0,000 y 0,001 respectivamente.

Entre las comorbilidades que presentan complicaciones destacan por su significación: la Insuficiencia cardiaca congestiva $(0,019)$; la Alteración de fluidos y electrolitos corporales $(0,040)$ y Anemia por otras deficiencias $(0,001)$. Del total de pacientes estudiados (481), el $15 \%$ no presentó comorbilidades. La media de comorbilidades entre los complicados es $2,36(1,49)$ y la significación intergrupos es de 0,006 . Los porcentajes más altos de complicación se dieron en pacientes con tres comorbilidades asociadas.

\section{DISCUSIÓN}

El estudio realizado pone de manifiesto la presencia de complicaciones producidas en pacientes mayores de 65 años ingresados en un hospital de tercer nivel del occidente andaluz, con diagnóstico principal de fractura de cadera.

En los pacientes estudiados la media de edad, de 83,16 , es superior a la media nacional de pacientes con fractura de cadera, de $79^{(2)}$, y a la mayoría de estudios fuera de España ${ }^{(19,20)}$, aunque la comparación resulta en muchos casos difícil, pues en aquellos estudios en que se indica la edad mínima de los sujetos para su inclusión, ésta varía desde los 50 hasta los 75 años.

Según el estudio realizado en Alemania por Eschbach DA et al. ${ }^{(22)}$, la mayoría de los pacientes con fractura proximal de fémur están en la octogésima década, lo que coincide con nuestro $57,6 \%$ de fracturas en pacientes con esas edades.

El porcentaje de mujeres del presente estudio, $77,75 \%$, está por encima de los dos tercios del total de pacientes, al igual que la gran mayoría de los estudios revisados.

El porcentaje de exitus, 4,99\%, se encuentra entre lo habitual del 2 al $7 \%$ de fallecidos durante la fase hospitalaria aguda ${ }^{(2)}$, o entre el 1,44 y $20 \%$ que es lo habitual según otros estudios ${ }^{(19)}$.

La estancia media de 10,2 días está cercana a las medias más bajas encontradas en la literatura revisada ${ }^{(21-23,25,26)}$, aunque no es fácil la comparación con otros teniendo en cuenta que en algunos casos se incluye en la estancia media los primeros días de rehabilitación. 
El diagnóstico más frecuente, fractura de sección trocanterea neom de cuello de fémur cerrada, es una fractura extracapsular, lo que está en consonancia con lo revisado ${ }^{(2,19,21-24)}$. La estancia media preoperatoria, de 3,5 días, es acorde con la mediana en España que se sitúa alrededor de los 3 días.

El tratamiento quirúrgico usado en las fracturas extracapsulares es la osteosíntesis, que, de forma lógica, es el tratamiento más utilizado en este estudio y en los revisados.

Respecto a las complicaciones, según el CCS, la complicación más frecuente es "complicaciones quirúrgicas-vías urinarias", sólo de acuerdo con algunos estudios ${ }^{(3,25)}$, aunque en la mayoría está entre las más frecuentes ${ }^{(2,8,19,26)}$.

Se han identificado un máximo de dos complicaciones por episodio. El 8,7\% de pacientes complicados, incluyendo los fallecidos, es mucho menor que el de la mayoría de estudios ${ }^{(25,26)}$, incluso aquellos que se centran únicamente en la complicaciones médicas, y que van del 14 al $20 \%{ }^{(26)}$. Esto puede ser debido a la limitación del estudio al coger datos del CMBD que pueden tener errores de codificación o estar incompletos.

En nuestro estudio, el sexo masculino y la edad más avanzada parecen estar ligados a las complicaciones, así como la demora quirúrgica, en consonancia con lo publicado por González-Montalvo Jl et al. ${ }^{(8)}$; no obstante, se opone a lo publicado por Eschbach DA et al. (22), que considera que la edad avanzada no está asociada a mayor incidencia de complicaciones, y con lo publicado por Kim SD et al. ${ }^{(27)}$, que afirman que el retraso en la cirugía no incrementa las complicaciones postoperatorias.

La relación entre la mayor estancia media en pacientes con complicaciones y las complicaciones podría indicar mayor riesgo de complicaciones ante un período de hospitalización más prolongado, o bien, que éste se prolongue por la aparición de complicaciones.

Se confirma que la presencia de comorbilidades guarda una relación significativa con las complicaciones. Las comorbilidades que han sido asociadas con la presencia de complicaciones en nuestro estudio son la "insuficiencia cardíaca congestiva", la "alteración de fluidos y electrolitos corporales" y la "anemia por otras deficiencias". La enfermedad cardiovascular ya ha sido relacionada con la aparición de complicaciones ${ }^{(28)}$.

La comparación de los resultados obtenidos en nuestro estudio en relación a la comorbilidad presente al ingreso y su relación con las complicaciones no es posible, pues la mayoría de la literatura revisada utiliza la clasificación de la American Society of Anesthesiologist (ASA) para recoger la comorbilidad, y en el presente se ha utilizado, como se indicó previamente, la comorbilidad según el índice de Elixhauser en pacientes con fractura de cadera.

\section{CONCLUSIONES}

La presencia de complicaciones en el período de hospitalización de pacientes ancianos ingresados por fractura de cadera se relaciona con sexo masculino, edad avanzada y demora quirúrgica. 
Esto tiene como implicación para la practica la necesidad de reducir la demora quirúrgica, que conlleva un aumento en el período de hospitalización, que puede a su vez incrementar el número de complicaciones y el gasto sanitario, así como la importancia de identificar a las personas con varias comorbilidades presentes al ingreso, para tratar de minimizar al máximo el número de complicaciones que puedan sufrir, pues parecen tener una mayor predisposición, y la pertinencia de instaurar vías clínicas estandarizadas para manejar estos pacientes y poder reducir así complicaciones, como las relacionadas con las vías urinarias.

\section{REFERENCIAS}

1. Instituto de Información Sanitaria. Estadísticas Comentadas: La Atención a la Fractura de Cadera en los Hospitales del SNS [Publicación en Internet]. Madrid: Ministerio de Sanidad y Política Social; 2010.

2. Monte-Secades $R$, Peña-Zemsch $M$, Rabuñal-Rey $R$, Bal-Alvaredo $M$, Pazos-Ferro A, Mateos-Colino A. Factores de riesgo para la presentación de complicaciones medicas en enfermos con fractura de cadera. Rev Calid Asist. 2011; 26(2): 76-82.

3. Martínez Ruiz A, Gómez Jorge C, Navarro Navarro R, Ruiz Caballero JA, Jiménez Díaz JF, Brito Ojeda ME. Complicaciones de fracturas de cadera en el anciano. XVI Jornadas Canarias de Traumatología y Cirugía Ortopédica; 2002. p. 89-91.

4. Alarcón T, González-Montalvo Jl. Fractura de cadera en el paciente mayor. Rev Esp Geriatr Gerontol. 2010; 45(3): 167-170.

5. Instituto Nacional de Estadística. Movimiento Natural de la Población e Indicadores Demográficos Básicos. Datos definitivos de 2011 y avance del primer semestre de 2012. Instituto Nacional de Estadística, Notas de Prensa, 19 de diciembre de 2012. Disponible en: http://www.ine.es/prensa/np759.pdf Consultado el 20 de mayo de 2013.

6. Servicio Andaluz de Salud. Examen de Salud para mayores de 65 años. Andalucía: Servicio Andaluz de Salud, Consejería de Salud, Junta de Andalucía; 2008.

7. Andalucía. Fractura de cadera en el anciano: Proceso Asistencial Integrado. Andalucía: Consejería de Salud, Junta de Andalucía; 2002.

8. González-Montalvo JI, Alarcón T, Hormigo Sánchez Al. ¿Por qué fallecen los pacientes con fractura de cadera?. Med Clin (Barc). 2011; 137(8): 355-360.

9. World Health Organization (WHO). Más que palabras: Marco Conceptual de la Clasificación Internacional para la Seguridad del Paciente. WHO; 2010. Disponible

en:

http://www.who.int/patientsafety/implementation/icps/icps full report es.pdf Consultado el 21 de mayo de 2013.

10. Sociedad Andaluza de Calidad Asistencial (SADECA). Recomendaciones para la seguridad del paciente en centros sanitarios. SADECA; 2012.

11. Reyes Revuelta JF. Taxonomía en seguridad del paciente: ¿Hablamos todos el mismo idioma?. Revista Páginasenferurg. 2011; 3 (10): 12-16.

12. WHO. Curso virtual de introducción a la Investigación en Seguridad del Paciente. Disponible en: www.who.int/patientsafety/research/Sesion1.pdf . Consultado el 21 de mayo de 2013.

13. Andalucía. Estrategia para la seguridad del paciente. Andalucía: Consejería de Salud, Junta de Andalucía; 2006. 
14. Aranaz-Andrés J, Limón-Ramírez R, Aibar-Remón C, Miralles-Bueno JJ, Vitaller-Burillo J, Terol-García E et al. Luces y sombras en la seguridad del paciente: estudio y desarrollo de estrategias. Informe SESPAS 2008. Gaceta Sanitaria 2008; 22 (1 Supl 4) : 198-204.

15. de Vries EN, Prins HA, Crolla RM, den Outer AJ, van Andel G, van Helden $\mathrm{SH}$ et al. Effect of a comprehensive surgical safety system on patient outcomes. N Engl J Med. 2010; 363(20): 1928-37.

16. Ministerio de Sanidad y Consumo. Estudio Nacional sobre los Efectos Adversos ligados a la Hospitalización. ENEAS 2005. Madrid: Ministerio de Sanidad y Consumo ; 2006.

17. World Health Organization. WHO Draft Guidelines for Adverse Event Reporting and Learning Systems. WHO: World Alliance for patient safety. Disponible

http://www.who.int/patientsafety/events/05/Reporting Guidelines.pdf Consultado el 29 de Mayo de 2013.

18. Andalucía. Manual de instrucciones del Conjunto Mínimo Básico de Datos de Andalucía. Año 2013. Disponible en: http://www.juntadeandalucia.es/servicioandaluzdesalud/library/plantillas/externa .asp?pag=...../publicaciones/datos/559/pdf/2013ManualCMBD.pdf Consultado el 20 de mayo de 2013

19. Brown CA, Boling J, Manson M, Owens T, Zura R. Relation between prefracture characteristics and perioperative complications in the elderly adult patient with hip fracture. South Med J. 2012;105(6):306-310.

20. Simunovic N, Devereaux PJ, Sprague S, Guyatt GH, Schemitsch E, Debeer $\mathrm{J}$ et al. Effect of early surgery after hip fracture on mortality and complications: systematic review and meta-analysis.CMAJ. 2010;182(15):16091616.

21. Wagner P, Fuentes $P$, Diaz A, Martinez F, Amenabar P, Schweitzer D et al. Comparison of complications and length of hospital stay between orthopedic and orthogeriatric treatment in elderly patients with a hip fracture. Geriatr Orthop Surg Rehabil. 2012;3(2):55-58.

22. Eschbach DA, Oberkircher L, Bliemel C, Mohr J, Ruchholtz S, Buecking B. Increased age is not associated with higher incidence of complications, longer stay in acute care hospital and in hospital mortality in geriatric hip fracture patients. Maturitas. 2013;74(2):185-189.

23. Rodríguez-Fernandez P, Adarraga-Cansino D, Carpintero P. Effects of delayed hip fracture surgery on mortality and morbidity in elderly patients. Clin Orthop Relat Res. 2011;469(11):3218-3221.

24. Sánchez-Crespo MR, Bolloque R, Pascual-Carra A, Pérez-Aguilar MD, Rubio-Lorenzo M, Alonso-Aguirre MA et al. Mortalidad al año en fracturas de cadera y demora quirúrgica. Rev esp cir ortop traumatol. 2010;54(1):34-38.

25. Poh KS, Lingaraj K. Complications and their risk factors following hip fracture surgery. J Orthop Surg (Hong Kong). 2013;21(2):154-157.

26. Merchant RA, Lui KL, Ismail NH, Wong HP, Sitoh YY. The relationship between postoperative complications and outcomes after hip fracture surgery. Ann Acad Med Singapore 2005;34(2):163-168.

27. Kim SD, Park SJ, Lee DH, Jee DL. Risk factors of morbidity and mortality following hip fracture surgery. Korean J Anesthesiol. 2013;64(6):505-510.

28. Roche JJ, Wenn RT, Sahota O, Moran CG. Effect of comorbidities and postoperative complications on mortality after hip fracture in elderly people: prospective observational cohort study. BMJ. 2005; 331(7529):1374. 
Recibido: 26 de enero 2014; Aceptado: 24 de abril 2014 